\title{
COMPORTAMIENTO IN VITRO DE CALLOS DE Dendranthema sp. (ASTERA- CEAE) EN MEDIO BASAL MS MODIFICADO
}

\author{
THE IN VITRO BEHAVIOUR OF THE CALLUS OF Dendranthema sp. (ASTERACEAE) IN MODI- \\ FIED BASAL MS MEDIUM
}

Rodolfo López $\mathrm{F}^{1}$, Anayibe Urriago $\mathrm{V}^{2}$, Diana E. Orozco R², Jorge L. Manjarrés $\mathrm{Ch}^{1}$.

$\begin{array}{r}\text { 1 Laboratorio de Biotecnología-CIBUQ. Universidad del Quindío, Colombia. } \\ \text { rlopez@uniquindio.edu.co } \\ 2 \text { Programa de Química. Universidad del Quindío, Colombia. } \\ \text { anyis7@hotmail.com, diana_e1234@hotmail.com. }\end{array}$
Recibido: Septiembre 3 de 2012
Aceptado: Octubre 19 de 2012
*Correspondencia del autor. Laboratorio de Biotecnología-CIBUQ. Universidad del Quindío, Carrera 15 Calle 12 Norte, Armenia,
Quindío, Colombia. rlopez@uniquindio.edu.co

\section{RESUMEN}

En la presente investigación se evaluó el desarrollo morfológico in vitro, de callos obtenidos a partir de segmentos nodales de plantas juveniles de Dendranthema sp., los cuales se dispusieron en medio basal MS modificado en sus macrosales y microsales, para determinar el medio basal MS modificado más adecuado para su crecimiento; se aplicaron seis tratamientos donde variaron las concentraciones de macrosales y microsales, a la mitad o al doble de ellas, en diferentes proporciones; los envases se sirvieron con $20 \mathrm{~mL}$ de medio de cultivo el cual estuvo suplementado con $1.0 \mathrm{mg} / \mathrm{L}$ de AIA y $3 \%$ de sacarosa; como agente gelificante se utilizó el producto comercial Culturegel a razón de $2.8 \mathrm{~g} / \mathrm{L}$ de medio preparado. Los mejores resultados se alcanzaron con el tratamiento Mitad-Mitad de relación macrosales-microsales, mientras que el tratamiento Mitad-Doble mostró los menores índices de crecimiento morfológico de los callos.

Palabras clave: Dendranthema sp., In Vitro, Macrosales, Microsales, Callogénesis, AIA.

\begin{abstract}
In the present study we evaluated the morphological development in vitro, callus obtained from nodal segments of young plants of Dendranthema grandiflorum, which were placed in modified MS basal medium in Microsales macrosales and to determine the most modified MS basal medium suitable for growth; six treatments were applied where macrosales varied concentrations and Microsales, half or twice them in different proportions were served containers with $20 \mathrm{~mL}$ of culture medium which was supplemented with $1.0 \mathrm{mg} / \mathrm{L}$ IAA and 3\% sucrose; gelificacnte agent was used as the commercial product Culturegel a rate of $2.8 \mathrm{~g} / \mathrm{L}$ of medium prepared. The best results were achieved with treatment half-half macrosalts-microsalts relationship, while the double-half treatment showed the lowest growth rates morphological calluses.
\end{abstract}

Keywords: Dendranthema sp., In Vitro, Macrosales, Microsales, Callogenesis, AIA. 


\section{INTRODUCCIÓN}

El crisantemo o pompón Dendranthema sp. (Ramat.) Kitam es una planta que pertenece a la familia Asteraceae. Es una especie ornamental para maceta y flor cortada, con alta demanda en el mercado (1); sus flores son portadoras de algunos metabolitos secundarios que hacen parte del mecanismo de defensa químico, muy eficaz contra la herbivoría (2).

Es una planta perenne, con hojas lanceoladas, lobuladas y muy perfumadas, cuyas flores con hermafroditas son polinizadas por insectos. La planta se desarrolla óptimamente a un rango de temperaturas de $18-25^{\circ} \mathrm{C}$ y una humedad relativa de entre $60-70 \%$. Los brotes de crisantemo comienzan su floración cuando la longitud de los días es mayor de 9,5 horas, por lo que es necesario ponerle iluminación artificial durante la noche. La técnica de cultivo in vitro como método de micropropagación ha permitido obtener material vegetal libre de microorganismos, de tal manera que con la micropropagación se estimula la brotación de yemas axilares o apicales, donde se pueden originar nuevos individuos con características semejantes a sus plantas madre (3). Por tanto, mediante la técnica de cultivo in vitro se planteó evaluar el desarrollo morfológico de callos de Dendranthema sp., en medio basal MS modificado, para identificar el más adecuado en el crecimiento y desarrollo de sus callos.

Las estrategias para estimular el aumento de síntesis de metabolitos secundarios son varias, entre las que se cuenta la incidencia de las macrosales y microsales, vitaminas y fitohormonas en el medio de cultivo basal; estos componentes son suficientes para garantizar el metabolismo de las células que en condiciones de cultivo in vitro son generalmente heterótrofas. Algunos de los medios más comúnmente utilizados son el MurashigeSkoog (MS), Linsmaierskoog (LS), Gamborg (B5) y Schenk-Hildebrandt (SH) (4).

La callogenésis es el proceso de crecimiento que se caracteriza porque, a partir de fragmentos de tejidos $\mathrm{u}$ órganos, se produce un tejido sin estructura específica que contiene un número limitado de algunos tipos de células especializadas encontradas en una planta intacta. Un tejido desorganizado (o callo) puede crecer con subcultivos y puede ser mantenido en medio sólido o líquido durante varios meses $(5,6,7,8)$.

El término callo corresponde a un conjunto de células procedentes de la desorganización de un tejido o de una suspensión de células; el callo tiene la peculiaridad de presentar células no diferenciadas para su última función pero que conservan el poder totipotente de dividirse, a manera de célula meristemática o embriogénica (6).

La más importante característica de los callos, desde el punto de vista funcional, es su capacidad para formar raíces, tallos y embriones capaces de regenerar plantas completas (7). Además, el cultivo de tejidos vegetales permite producir compuestos con actividad biológica de interés para el hombre, con gran número de metabolitos secundarios (9).

Para diferentes variedades de Dendranthema sp., se cuenta con variados reportes de inducción de callogénesis en crisantemo, en los cuales se evaluó diferentes tipos de explantes y la combinación de diversos reguladores hormonales $(8,10,11,12,13)$.

Desde el punto fitoquímico, algunas plantas se cultivan para la extraccion de metabolitos secundarios, por ejemplo el Arbol del Neen (Azadirachta indica A. Juss), se cultiva para la extraccion de Azadiracta (AZa), un metabolito secundario efectivo contra insectos acaros, nematodos, hongos y bacterias $(14,15,16)$.

Las plantas han sido desde la antigüedad un recurso al alcance del hombre para su alimentacion y la curación de sus enfermedades; en la actualidad cientos de plantas son utilizadas en la medicina, pero la ciencia moderna analizando y estudiando los efectos terapeuticos de las plantas quiere precisar, comparar y clasificar las diversas propiedades, con el fin de conocer los principios activos (metabolitos) responsables de eliminar o aliviar enfermedades .

Con respecto a la nutrición in vitro, Ramage y Williams (19) reportaron que la formación de callos de Nicotiana tabacum presentó limitaciones cuando evaluaron la ausencia de varios elementos minerales en el medio de cultivo; así, con ausencia de N no se logró la formación de callos; además, con la ausencia de $\mathrm{P}$, la formación de callos alcanzó sólo un 13.3\%, mientras que la ausencia de K permitió la formación de callos en un 33.3\%; la ausencia de $\mathrm{Mg}$ fue la que menos impacto causo cuando permitió la formación de $95.2 \%$ de callos. Sin embargo, incrementos en niveles de $\mathrm{P}_{2} \mathrm{O}_{5}$ pueden limitar la disponibilidad de otros iones minerales como $\mathrm{Fe}+$ y $\mathrm{Ca}+$. 


\section{MATERIALES Y MÉTODOS}

Para la presente investigación se tomaron callos inducidos en el Laboratorio de Biotecnología Vegetal-CIBUQ, a partir de segmentos nodales de Dendranthema sp. (8), los cuales se sometieron a multiplicación en medio basal MS, propuesto por Murashige y Skoog (20), suplementado con $1.0 \mathrm{mg}$ de la auxina AIA. Los callos se sembraron con un tamaño aproximado de $0.5+0.1$ $\mathrm{cm}$ de diámetro. Mediante un análisis descriptivo, el desarrollo morfológico de los callos, a las cuatro semanas de cultivo, fue de la siguiente manera:

Se realizó un diseño de bloques al azar con 20 repeticiones o bloques por tratamiento, en un arreglo factorial con dos factores controlados, en el medio de cultivo: a) macrosales con tres niveles (normal, doble y mitad); y b microsales con tres niveles (normal, doble, mitad), durante cuatro semanas de cultivo. La variable de respuesta consistió en crecimiento en diámetro del callo y en apariencia morfológica.

Las macrosales modificadas fueron: $\mathrm{NH}_{4} \mathrm{NO}_{3}, \mathrm{KNO}_{3}$, $\mathrm{KH}_{2} \mathrm{PO}_{4}, \mathrm{MgSO}_{4}$; las microsales modificadas: $\mathrm{H}_{3} \mathrm{BO}_{3}$, $\mathrm{MnSO}_{4}, \mathrm{ZnSO}_{4}, \mathrm{CuSO}_{4}, \mathrm{CoCl}_{2}, \mathrm{Na}_{2} \mathrm{MoO}_{4}$; los demás componentes del medio basal MS permanecieron constantes. El pH de la solución se ajustó a $6.0+2.0$; cada envase se sirvió con $20 \mathrm{~mL}$ de medio basal para ser llevados a condiciones de autoclave $\left(121^{\circ} \mathrm{C}\right.$ y $\left.15 \mathrm{psi}\right)$ (Tabla 1).

Tabla 1. Diferentes concentraciones del medio basal MS: Normal (N), Doble (D), y Mitad (M), según el tratamiento a aplicar.

\section{TRATAMIENTO MACROSALES MICROSALES}

\begin{tabular}{lll}
\hline & & \\
2 & $\mathrm{M}$ & $\mathrm{M}$ \\
3 & $\mathrm{~N}$ & $\mathrm{M}$ \\
4 & $\mathrm{M}$ & $\mathrm{N}$ \\
5 & $\mathrm{~N}$ & $\mathrm{D}$ \\
6 & $\mathrm{M}$ & $\mathrm{D}$ \\
$\mathrm{H}$ & $\mathrm{D}$ & $\mathrm{M}$ \\
\hline
\end{tabular}

\section{RESULTADOS Y DISCUSIÓN}

Luego de cuatro semanas de cultivo en sala de crecimiento, los callos presentaron las siguientes características de acuerdo a los tratamientos aplicados, así:

T1. Mitad/mitad: El promedio de los callos de Den- dranthema sp. creció el doble de su original; en este tratamiento, los callos presentaron aspecto fresco y friable, con color verde suave a intenso; los callos mostraron mejor aspecto morfológico y mayor crecimiento que en los demás tratamientos (Figura 1). Aunque para Ramage y Williams (15), la ausencia total de $\mathrm{N}$ impide el crecimiento de masa callogénica, se puede interpretar en la medida que bajo contenido de $\mathrm{P}$ (macronutriente) permite la disponibilidad de iones como $\mathrm{Fe} \mathrm{y} \mathrm{Ca}$.

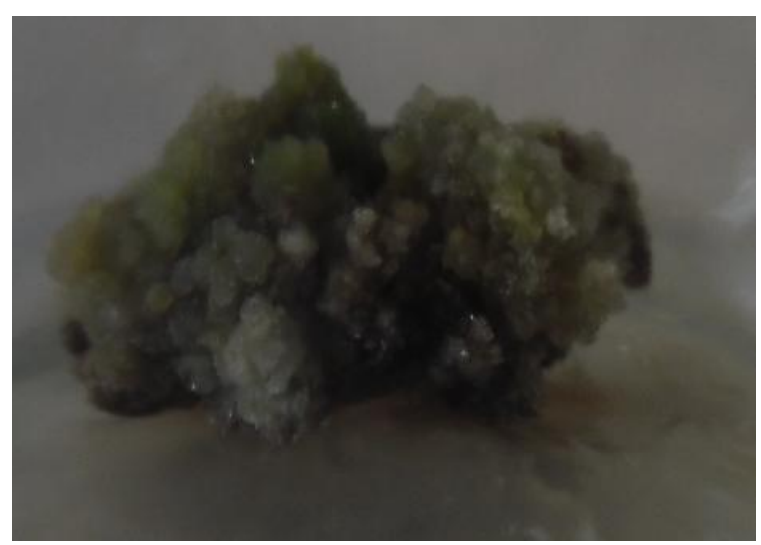

Figura 1. Callo sembrado en medio basal MS modificado (macrosales:mitad- microsales:mitad).

T2. Normal/mitad: El promedio de los callos de Dendranthema sp. incrementó su tamaño en una cuarta parte en relación con su tamaño inicial; la seccion en la que el callo habia sido cortado en su multiplicación, presentó regeneracion de nuevo callo y su color verde claro se hizo muy evidente.

T3. Mitad/normal: Los callos de Dendranthema sp. se observaron sanos y con algunas zonas de color verde y otras blancas, con poca ganancia en crecimiento (Figura 2).

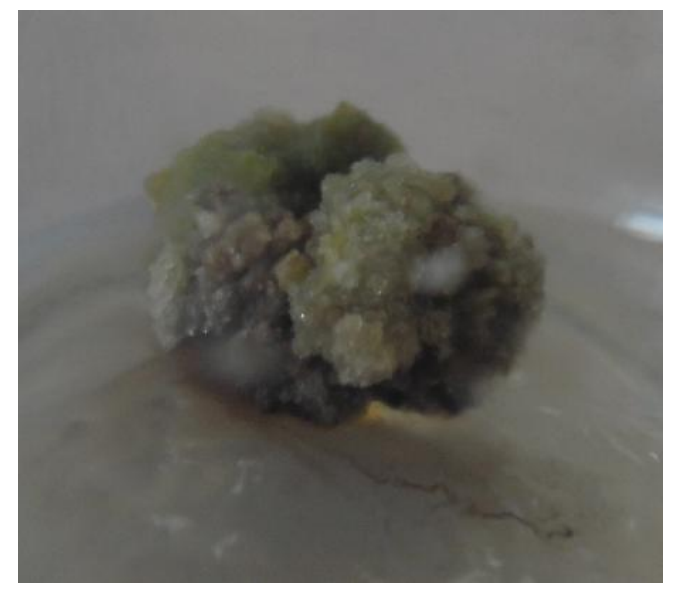

Figura 2. Callo sembrado en medio basal MS modificado (macrosales:mitad- microsales:normal). 
Normal/doble: El promedio del tamaño de los callos de Dendranthema sp. aumentó en una cuarta parte, en relación con su tamaño inicial, mostrando coloracion pálida en algunos callos.

Mitad/doble: Los callos de Dendranthema sp. inoculados en este tratamiento no exhibieron aumento del tamaño, con aspecto poco satisfactorio ( Figura 3).

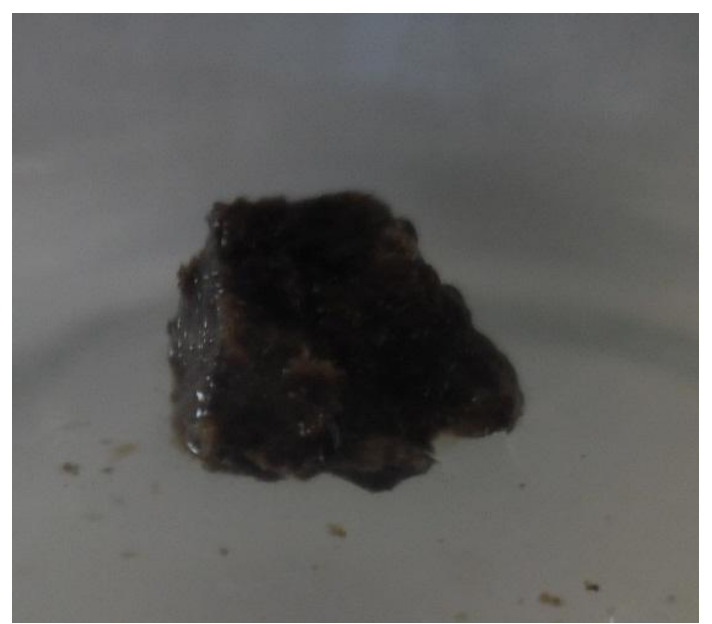

Figura 3. Callo sembrado en medio basal MS modificado (macrosales:mitad- microsales:doble).

Doble /mitad: El promedio de los callos de Dendranthema sp. mostró un crecimiento cercano al $25 \%$ de su tamaño original, con coloracion verde clara, aunque algunos presentaron coloracion amarillenta en su parte basal (Figura 4).

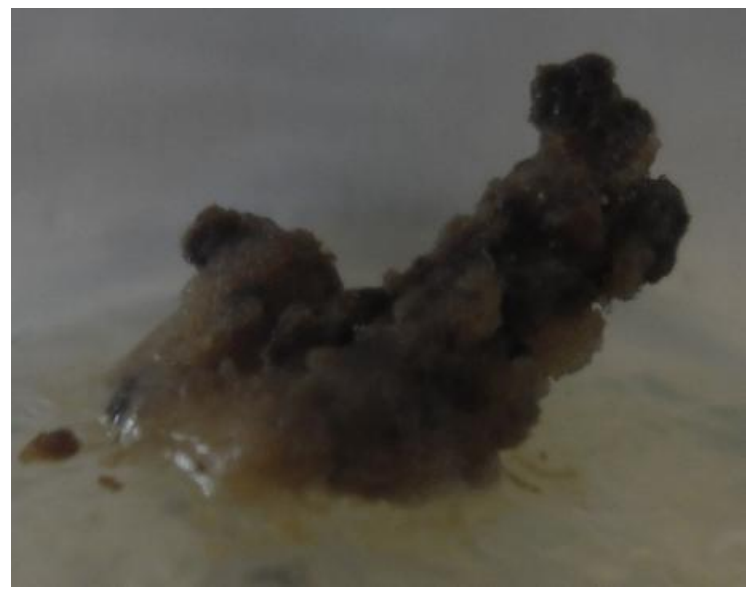

Figura 4. Callo sembrado en medio basal MS modificado (macrosales:doble- microsales:mitad)

\section{CONCLUSIONES}

El mejor comportamiento de los callos de Dendranthema sp. multiplicados in vitro, se logró cuando crecieron en medio basal MS modificado a la mitad de concentraciones de las macrosales y las microsales, mientras que los callos que crecieron en medio basal MS modifcado en las macrosales a la mitad y las mirosales en concentración doble, no lograron aumento en tamaño en crecimiento, indicando el desarrollo más pobre de los tratamientos . 


\section{BIBLIOGRAFIA}

1. Enríquez Del Valle, J.R, Velásquez Toro B, Vallejo Fuentes A.R, Velasco Velasco V. A. Nutrición de Plantas de Dendranthema grandiflorum, obtenidas in vitro durante su aclimatación en invernadero. Revista fitotécnica Mexicana. Vol. 28. N004.Sociedade Mexicana de Fitogenética, A.C. Chapingo México. 2005. Pág.377-383.

2. Anaya L. Ecología química. México. P y V editores. 2003. pp 140.

3. López, F. Micropropagación vegetal Una guía teórica práctica. Editorial Arte Imagen. 2009. 80p.

4. Arias Sabala M, Angarita Velasquez, Aguirre Cardona A.M, Restrepo Florez J.M, Montoya Vallejo C. Estrategias para incrementar la producción de Metabolitos secundarios en cultivos de Células vegetales. 2009. Pág. 4081-4895.

5. Reynier A. Gonzales J.A. Manual de viticultura. España. Mundi prensa. 2002.

6. Abdelnour A. Escalant J.V. Conceptos básicos del cultivo de tejidos vegetales. Catie. Costa rica. 1994.

7. Gonzáles O. Silva JJ. Espinosa A. Ros C. Hernández MM. La embriogénesis somática en Ipomoea: una posibilidad para la multiplicación y conservación de los recursos naturales. Granma. 2012.

8. Manjarrés J. L. establecimiento in vitro de crisantemo Dendranthema grandiflorum (Ramat.) Kitam, variedades white polaris y golden polaris. Trabajo de Grado como Biólogo. Programa de Biología. Universidad del Quindío. Colombia. 2011. 63p.

9. May R A. y Trigiano RN. Somatic embryogenesis and plant regeneration from leaves of Dendranthema grandiflora. J. Am. Soc. Hort. Sci. 1991. 116: 366-371.

10. Pavingerová D, Dostál J, Bísková R Y Benetka V. Somatic embryogenesis and Agrobacterium-mediated transformation of chrysanthemum. Plant Sci. 1994. 97: 95-101

11. Urban LA, Sherman JM, Moyer JW Ydaub ME. High frequency shoot regeneration and Agrobacterium-mediated transformation of chrysanthemum (Dendranthema grandiflora). Plant Sci. 1994. 98: 69-79

12. Teixeira Da Silva JA. Control of chrysanthemum organogenesis by thin cell layer technology. Asian J. Plant Sci. 2003 a. 2: 505-514

13. Santos MS. Velásquez Y. González MM. Producción de pigmentos por callos de Mammillaria candida sheidweiler (Cactaceae). Red de revistas científicas de América latina y el Caribe, España y Portugal. México. 2005. 619-626.

14. National research Council (NRC). Neem a tree for solving global problems report of an ad Hoc panel of 25 the board on sci. and technol for international development national Academy press Washington, D.C. 1992.107 p.

15. Gera M, N Gera, SL Menna, T Singh. vanation in response in ten provenances of Azadirachta indica A. Juss. The indicant forester. 1998. 124 (9):696-701

16. Herrera P. Villegas M. López R. Evaluación de la respuesta morfogénica y tamizaje fitoquímico de callo obtenido a partir de disco de hoja Azadirachta indica A. Juss (Meliaceae). Universidad del Quindío. Armenia. 2011. pp.100-112.

17. Sanabria A. Análisis fitoquímico preliminar. Bogotá. 1983.

18. Molina L, Gómez M. Castaño J. Contribución al estudio fitoquímico de la especie Sanseviera trifasciata Prain y su comportamiento frente a ensayos biológicos. Revista de Investigaciones, Universidad del Quindío. Armenia. 2011. N²2: 121-122.

19. Ramage C., Williams R. Mineral nutrition and plant morphogenesis. In Vitro Cell. Dev. Biol.-Plant. 2002. 38:116-124.

20. Murashige T, Skoog F. A revised medium for rapid growth and bioassays with tobacco tissue culture. Physiol. Plant. 1962. 15: 473-479. 